\title{
IN VIVO IMAGING USING BIOLUMINESCENCE FOR EVALUATION OF ANTITUMORAL EFFICACY IN A BREAST CANCER MODEL
}

Raquel S. Faria', Luiza I. de Lima¹, Marina S. Franco², Marjorie C. Roque², Mônica C. Oliveira², Ricardo B. Azevedo', João Paulo F. Longo'

${ }^{1} G e n e t i c s$ and Morphology Department, Institute of Biological Science, Universidade de Brasília - Brasília (DF), Brazil. ${ }^{2}$ Department of Pharmaceutical Products, Faculty of Pharmacy, Universidade Federal de Minas Gerais - Belo Horizonte (MG), Brazil.

Objective: To evaluate the Antitumor activity of a liposomal nanosystem containing Paclitaxel and Doxorrubicina (pHSL-PTX/DXR), by monitoring the tumor cells bioluminescence in vivo. Methodology: The tumor cell line 4T1 (adenocarcinoma mammary murine - ATCC CRL-2539) was modified using lentivirus particles Lentifect ${ }^{\text {win }}$ (GeneCopoeia, MD, EUA), according to the manufacturer's protocol. The cells were cultivated in DMEM medium, with fetal bovine serum and antibiotics, $10 \%$ and $1 \%$, respectively. The orthotropic implants of 4T1-luc $\left(1 \times 10^{5}\right)$ was performed in the left fifth breast of female Balb/c mouse. Throughout the procedure, the animals were kept under inhalation anesthesia with isoflurane (2.5\%). The treatment strted after 14 days of tumor induction, in the following groups: $p H S L-P T X / D X R, p H S L$ -DXR; pHSL-PTX, free DXR, free PTX e free PTX/free DXR. The intravenous treatments were performed in 4 doses of 100 $\mu \mathrm{l}$, three days interval. For the bioluminescence images, $100 \mu \mathrm{L}$ of D-Luciferin (concentration $150 \mathrm{mg} \cdot \mathrm{kg}^{-1}$ ) (Sigma) was used. The animals were kept under anesthesia in the Lumina XR IVIS equipment, with 1 minute exposure time. The quantification of the photon signal was processed in the Living Image 3.0 program, Caliper Life Sciances, Alameda, CA. Results: The $p H S L-P T X / D X R$ treated group system showed the smallest sign of bioluminescence during and after treatment, showing a reduction in tumor mass, and consequently better efficacy against primary breast tumor. There was also a reduction of the light signal in the lung region, indicating a decrease in the metastasis process caused by this type of tumor cell. Conclusion: using the in vivo bioluminescence method it was possible to evaluate the antitumor efficacy of the liposomal nanosystem $p H S L-P T X / D X R$, and the non-invasive monitoring of pulmonary metastases. 